\title{
High five
}

$5 G$ is our 2020 technology of the year.

ast January, we launched our technology of the year and chose edge computing as our pick for 2019. Reflecting the thoughts of the editorial team, our aim with this technology feature is to highlight an area that we think has achieved a key breakthrough or reached an important moment of development, be it fundamental or applied, be it in academia or industry. For 2020, we have chosen $5 \mathrm{G}$ - the fifth generation of wireless communications technology.

A new generation of wireless network has appeared approximately every ten years: the first $(1 \mathrm{G})$, which was an analogue system and focused on voice calls, emerged in around 1980; the second (2G), which saw the switch to digital and provided data services such text messaging, emerged in around 1990; the third (3G), which offered Internet access and e-mail, emerged in around 2000; and the fourth (4G), which made video calls mainstream, emerged in around 2010. 5G has been in development for a number of years now and deployment is already underway. But the technology - and the applications it could open up are just getting started. 2020 is the year we expect to see it start to make a difference.

The technology promises much, including higher data rates and more reliable communication. And for future applications that are reliant on connectivity - from the Internet of Things (IoT) to driverless cars to augmented reality - its capabilities should be central. In the first of a series of articles in this issue on the topic, Muriel Médard of Massachusetts Institute of Technology explores the technology behind these capabilities, and how it differs from 4G. Then, in a related Comment article, Vida Ilderem of Intel Labs highlights the fact that work on $5 \mathrm{G}$ is far from done, and that innovation from the component to the system level will be required in order for it to deliver its full potential.

$5 \mathrm{G}$ technology is expected to have the capacity and reliability to support digital healthcare applications, including remote consultations and robotic telesurgery. It could also be cheaper to deploy and maintain than previous generations. Thus, and as Joyce Mwangama, Bessie Malila,

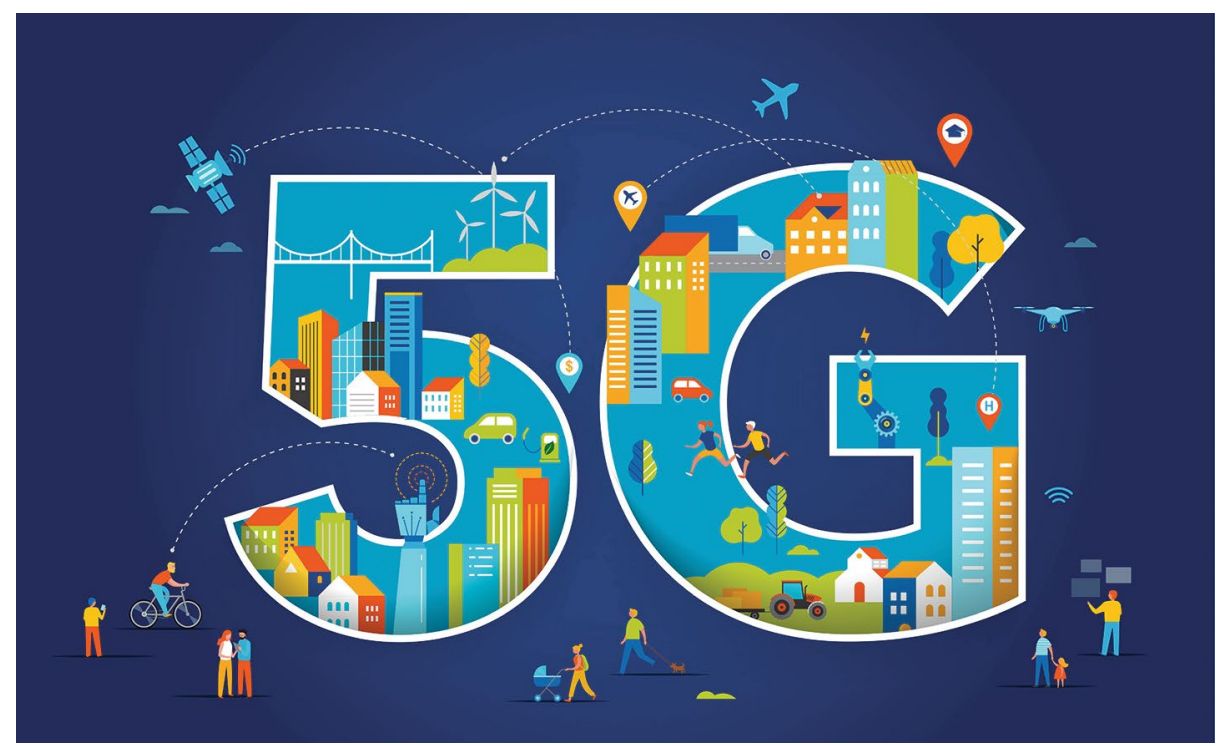

Credit: Marina Zlochin / Alamy Stock Vector

Tania Douglas and Molebogeng Rangaka of the University of Cape Town and University College London explain in a Comment article elsewhere in this issue, it could provide a route for the development of digital healthcare in rural Africa.

The significance of $5 \mathrm{G}$ also has a political dimension. Governments are concerned about control of the $5 \mathrm{G}$ infrastructure and the US has, in particular, effectively banned Chinese suppliers of the necessary network equipment, including Huawei. In a further Comment article, Paul Timmers of the University of Oxford argues that governments have underestimated the importance of technology governance in the development of 5G. As a result, they will now look to reassert control over key technologies and standards, a realignment that could affect technologies beyond 5G, including a potential sixth generation of wireless network (6G), IoT and artificial intelligence. Therefore, if these technologies are to be based on global standards, and are to avoid becoming geographically fragmented, industry consortia and the global technology community need to address this issue.
The $5 \mathrm{G}$ era has only just begun, but for some researchers, thoughts have already turned to the question of what is next. In a Perspective in this issue, Shuping Dang, Osama Amin, Basem Shihada and Mohamed-Slim Alouini of the King Abdullah University of Science and Technology in Saudi Arabia provide a vision for what $6 \mathrm{G}$ could be. They suggest that it should be human centric (as opposed to machine, application or data centric) and that security, secrecy and privacy should be the key attributes. This vision is built on a systematic framework in which Dang and colleagues anticipate potential application scenarios for $6 \mathrm{G}$ and explore the required communication technologies.

Whether this or any other vision of $6 \mathrm{G}$ emerges will depend on a variety of factors, some scientific and technological, others economic, social and political. Something to watch out for in our 2030 technology of the year, perhaps.

Published online: 24 January 2020 https://doi.org/10.1038/s41928-020-0368-1 